\title{
Baicalein inhibits migration and invasion of gastric cancer cells through suppression of the TGF- $\beta$ signaling pathway
}

\author{
FENGLIN CHEN $^{1}$, MINGKAI ZHUANG ${ }^{2}, \mathrm{JUN} \mathrm{PENG}^{3}, \mathrm{XIAOZHONG} \mathrm{WANG}^{1}$, TINGXUAN HUANG $^{4}, \mathrm{SANMEI} \mathrm{LI}^{1}$, \\ MANQIANG LIN ${ }^{1}$, HONGMING LIN ${ }^{1}$, YATING XU ${ }^{1}$, JIANYING LI ${ }^{1}$, ZHIXIN CHEN $^{1}$ and YUEHONG HUANG ${ }^{1}$ \\ ${ }^{1}$ Department of Gastroenterology, Union Hospital of Fujian Medical University; ${ }^{2}$ College of Union Clinical Medicine, \\ Fujian Medical University; ${ }^{3}$ Academy of Integrative Medicine Biomedical Research Center, Fujian University of \\ Traditional Chinese Medicine; ${ }^{4}$ College of Basic Medical Sciences, \\ Fujian Medical University, Fuzhou, Fujian 350001, P.R. China
}

Received October 7, 2013; Accepted June 17, 2014

DOI: $10.3892 / \mathrm{mmr} .2014 .2452$

\begin{abstract}
The transforming growth factor- $\beta$ (TGF- $\beta$ ) signaling pathway exhibits an important role in cancer invasion and metastasis. Excessive expression of TGF- $\beta$ activates Smad4, leading to the upregulation of downstream metastasis-associated genes. Thus, the inhibition of the TGF- $\beta / \mathrm{Smad} 4$ signaling pathway may be a novel strategy for treatment of cancer metastasis. Baicalein, a flavonoid derived from the root of Scutellaria baicalensis, has been reported to exert strong anti-tumor activity towards various types of cancer. In the present study the effect of baicalein on migration and invasion of cancer cells was evaluated using wound-healing and Transwell assays. In order to investigate the possible molecular mechanisms of the anti-metastatic effects of baicalein, quantitative polymerase chain reaction (qPCR) and western blot analyses were performed to examine the effect on the expression of TGF- $\beta$, Smad4, N-cadherin, vimentin, ZEB1 and ZEB2. It was determined that baicalein inhibited the migration and invasion of AGS cells by suppressing the TGF- $\beta /$ Smad4 signaling pathway. In addition, baicalein treatment reduced the expression of the metastasis-associated $\mathrm{N}$-cadherin, vimentin, ZEB1 and ZEB2, downstream target genes of the TGF- $\beta / S m a d 4$ signaling pathway. Collectively, these results suggest that inhibition of the metastasis of cancer cells via inactivation of TGF- $\beta / \mathrm{Smad} 4$ signaling is one of the mechanisms by which baicalein may treat cancer.
\end{abstract}

Correspondence to: Dr Xiaozhong Wang, Department of Gastroenterology, Union Hospital of Fujian Medical University, 29 Xinquan Road, Fuzhou, Fujian 350001, P.R. China

E-mail: drwangxz@pub6.fz.fj.cn

Key words: baicalein, gastric carcinoma, neoplasm metastasis, transforming growth factor- $\beta$

\section{Introduction}

Gastric cancer (GC) is one of the most common causes of cancer-related mortality, with $>700,000$ mortalities worldwide every year (1). Although the prognosis of early GC is generally favorable, a large proportion of cases of GC are diagnosed in the locally advanced or disseminated stage. Chemotherapy remains an important therapeutic modality for patients with advanced GC. However, due to multi-drug resistance and adverse side-effects, the objective response to conventional treatments is not satisfactory in the majority of patients with metastatic GC. The overall 5-year survival rate of GC patients is only $20-25 \%$ (2). Therefore, more effort must be devoted to developing novel therapeutic or preventive strategies and/or agents that target cancer metastasis.

Transforming growth factor- $\beta$ (TGF- $\beta$ ) is a multifunctional cytokine that controls a number of different cell functions and regulates several important processes during tumor progression, including proliferation, apoptosis, angiogenesis, invasion, and metastasis (3). TGF- $\beta$ exerts a growth inhibition function in premalignant cells, but promotes tumor metastasis in later stages of cancer. Increased expression levels of TGF- $\beta$ have been detected in numerous types of tumor, including GC, and are correlated with the invasion and metastasis of GC; thus it is a potential marker for the identification of GC patients with a poor prognosis (4-6). Furthermore, TGF- $\beta$ treatment may increase the metastatic potential of the BGC823 and SGC7901 GC cell lines (7). This suggests that the TGF- $\beta$ signaling pathway has an important role in cancer invasion and metastasis and that its modulation may be a novel strategy for treatment of GC metastasis.

It has previously been reported that flavonoid compounds may have a potential inhibitory effect on cancer progression $(8,9)$. Of these substances, baicalein, a flavonoid derived from the root of Scutellaria baicalensis, exerted strong anti-metastatic activity against various types of cancer cell, including breast (10), skin (11), bladder (12), liver (13) and bone (14) cancers. However, the mechanisms by which baicalein exerts its anti-metastatic effects are largely unknown. The aim of the present study was to use the AGS human GC 
cell line to evaluate the effect of baicalein on the migration and invasion of cancer cells, and to investigate the possible molecular mechanisms of its anti-metastatic effects.

\section{Materials and methods}

Cell culture and baicalein treatment. The AGS human GC cell line was purchased from Nanjing KeyGEN Biotech. Co., Ltd. (Nanjing, China). Cells were cultured with RPMI1640 medium supplemented with $10 \%$ fetal bovine serum (FBS), $100 \mathrm{U} / \mathrm{ml}$ penicillin and $100 \mu \mathrm{g} / \mathrm{ml}$ streptomycin in a humidified incubator at $37^{\circ} \mathrm{C}$ under $5 \% \mathrm{CO}_{2}$ in air. Cell culture materials were purchased from Life Technologies (Grand Island, NY, USA). For baicalein treatment, an appropriate concentration of baicalein (Enzo, Farmingdale, NY, USA) was added to the culture medium to achieve the indicated concentrations and then incubated with cells for indicated time points. A dimethylsulfoxide solution (Solarbio, Beijing, China) without baicalein was used as the control.

Wound-healing assay. Cells were seeded into a 6-well plate and allowed to grow in complete medium until approximately confluent. Cell monolayers were wounded using a 250- $\mu 1$ tip and washed twice with phosphate-buffered saline to remove cell debris. The cells were then incubated in culture medium in the absence or presence of baicalein $(0,25$ or $50 \mu \mathrm{M})$ for $24 \mathrm{~h}$. Images of treated cells moving within the wound were captured using a Leica DM IL LED inverted microscope and the average width of the wound was analyzed at the indicated times by LAS software v.4.0 (Leica Microsystems GmbH, Wetzlar, Germany).

Cell invasion and migration assays. Transwell chambers with a $6.5-\mathrm{mm}$ diameter and an $8.0-\mu \mathrm{m}$ pore polycarbonate membrane (Corning Life Sciences, New York, NY, USA) were coated with Matrigel (20 $\mu \mathrm{g} / \mathrm{insert}$; BD Bioscience, Bedford, MA, USA) for $6 \mathrm{~h}$ to form a basement membrane prior to use. Following treatment with baicalein $(25$ or $50 \mu \mathrm{M})$ for $24 \mathrm{~h}$, the surviving cells were harvested and seeded in the upper chamber at a density of 30,000 cells/well in $200 \mu \mathrm{l}$ free-serum culture medium. Culture medium containing 10\% FBS $(600 \mu \mathrm{l})$ was added in the lower chamber as a chemoattractant. After incubation for $24 \mathrm{~h}$ at $37^{\circ} \mathrm{C}$, all of the non-invaded cells were removed from the upper face of the membrane with a cotton swab. The invaded cells on the lower face of membrane were then fixed for 10 min with $4 \%$ paraformaldehyde (Genmed Scientifics, Inc., Arlington, MA, USA) and stained with $0.2 \%$ crystal violet for $15 \mathrm{~min}$. Cells that had invaded through the membrane were counted using the inverted microscope (x200 magnification).

The general procedure of the migration assay was similar to that of the invasion assay, with the exception that the membranes in each chamber were not coated with Matrigel and the number of cells seeded in the upper chamber was 50,000 cells/well.

Quantitative polymerase chain reaction ( $q P C R)$. Following treatment with baicalein for $24 \mathrm{~h}$, total RNA was extracted from cells using RNAiso reagent (Takara, Dalian, China) according to the manufacturer's instructions. cDNA was synthesized

Table I. qPCR primers.

Sequence of primers $\left(5^{\prime}-3^{\prime}\right)$

TGF- $\beta$

Smad4

N-cadherin

Vimentin

ZEB1

ZEB2

GAPDH
S: CCGACTACTACGCCAAGGAG A: TGTGTACTCTGCTTGAACTTGTC

S: CATCTGAGTCTAATGCTACC

A: CAACAGTCCTTCACTATGG

S: AAGAACGCCAGGCCAAACAAC

A: CTGGCTCAAGTCATAGTCCTGGTCT

S: CCTCTTCCAAACTTTTCCTCCCT A: AAGTTTCGTTGATAACCTGTCCATC

S: AAGAATTCACAGTGGAGAGAAGCCA

A: CGTTTCTTGCAGTTTGGGCATT

S: TGTCATTAGAAGAGGCGTAA

A: GCAGAGCAGGTTAGAACT

S: CATCAGCAATGCCTCCTGCAC A: TGAGTCCTTCCACGATACCAAAGTT

qPCR, quantitative polymerase chain reaction; TGF- $\beta$, transforming growth factor- $\beta$; $S$, sense; $A$, antisense.

from $1 \mu \mathrm{g}$ total RNA using an PrimeScript ${ }^{\mathrm{TM}}$ RT Reagent kit (Takara). qPCR analysis was performed using SYBR ${ }^{\circledR}$ Select Master mix (Applied Biosystems, Foster City, CA, USA) with a 7500 Fast Real-Time PCR system with (v.2.0.5 software; Applied Biosystems). The following cycling conditions were used: 40 cycles of $50^{\circ} \mathrm{C}$ for $2 \mathrm{~min}, 95^{\circ} \mathrm{C}$ for $2 \mathrm{~min}, 58^{\circ} \mathrm{C}$ for $15 \mathrm{sec}$ and $72^{\circ} \mathrm{C}$ for $1 \mathrm{~min}$. The data were analyzed using the $2^{-\Delta \Delta C t}$ method and the ABI 7500 PCR system software (Applied Biosystems). The gene expression levels were normalized to GAPDH and presented as a relative fold change compared with the control. All reactions were independently repeated three times and the mean was calculated. The sequences of the primers used are presented in Table I.

Western blotting analysis. After treatment with baicalein for $24 \mathrm{~h}$, the cells were lysed in RIPA buffer (Pierce, Rockford, IL, USA) with Complete Protease Inhibitors and PhosSTOP Phosphatase Inhibitor Cocktail tablets (Roche, Mannheim, Germany). The lysates were then clarified by centrifugation at $4^{\circ} \mathrm{C}$ for $15 \mathrm{~min}$ at $30,270 \mathrm{x} \mathrm{g}$. The concentrations of the proteins in the supernatants were detected using a BCA Protein Assay kit (Pierce, Rockford, IL, USA). Equal amounts of the proteins were then separated by $10 \%$ SDS-PAGE gels and transferred onto the polyvinyl difluoride (PVDF) membranes (Roche). The PVDF membranes were blocked with $3 \%$ bovine serum albumin for $1 \mathrm{~h}$ and then probed overnight at $4^{\circ} \mathrm{C}$ with the primary antibodies listed below. Following three washes with Tris-buffered saline with Tween 20, the membranes were incubated with the appropriate horseradish peroxidase (HRP)-conjugated secondary antibody for $1 \mathrm{~h}$ at room temperature. The bands were detected using the Enhanced Chemiluminescent substrate (Pierce, Rockford, IL, USA). The primary antibodies used were as follows: N-cadherin (1:1,000, \#MA5-15633; Pierce), 
A

$\mathbf{0 ~ h}$

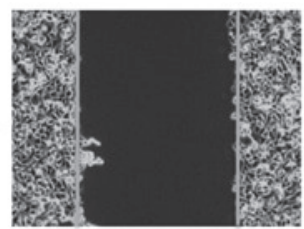

$24 \mathrm{~h}$

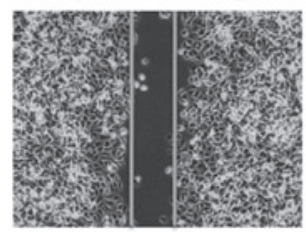

0
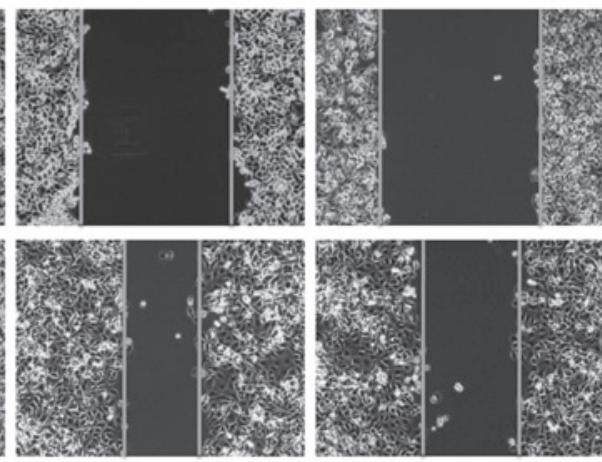

25

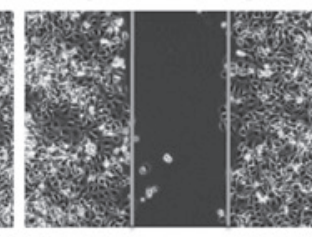

50
B

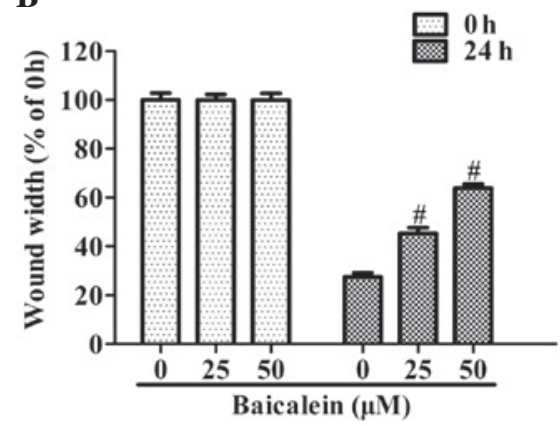

Baicalein $(\mu \mathrm{M})$

Figure 1. Inhibitory effect of baicalein on the motility of AGS cells. (A) Treatment with baicalein reduced the speed of wound healing in the AGS tissue (magnification, x100). (B) Quantification of the wound-healing assay. The data were normalized to the wound width of each group at 0 h. Data are represented as the mean \pm standard deviation of three separate experiments. ${ }^{\#} \mathrm{P}<0.05$ compared with untreated cells at the same time point.

A

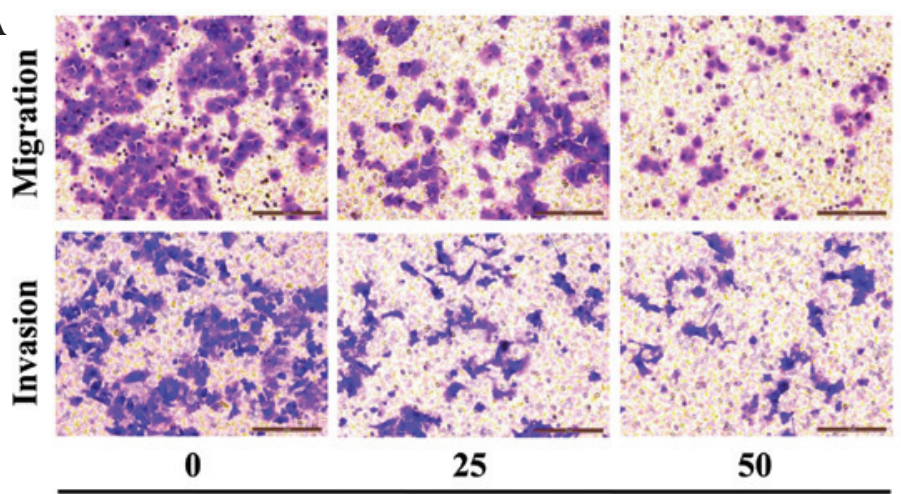

Baicalein $(\mu \mathrm{M})$

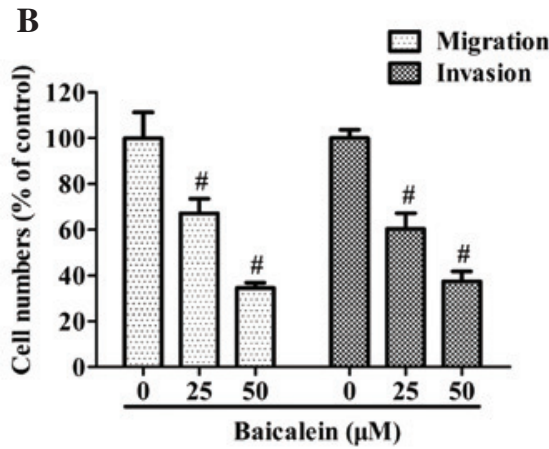

Figure 2. Inhibitory effect of baicalein on the migration and invasion of AGS cells. (A) Treatment with baicalein reduced the number of cells which migrated to or invaded the bottom side of the Transwell filter (magnification, x200). (B) Quantification of migration and invasion assay. The data were normalized to the migratory or invasive cell numbers of untreated group. Data are represented as the mean \pm standard deviation of three separate experiments. ${ }^{\#}<<0.05$ compared with untreated cells.

vimentin (1:1,000,\#21488; Signalway Antibody, College Park, MD, USA), ZEB1 (\#ab155249), ZEB2 (1:1,000, \#ab25837; Abcam, Hong Kong, China), TGF- $\beta$ (\#3709), Smad4 (\#9515) and $\beta$-actin (\#4967, 1:1,000; Cell Signaling Technology, Inc., Beverly, MA, USA). The following secondary antibodies used were: HRP-conjugated goat anti-mouse $\operatorname{IgG}$ and goat anti-rabbit IgG, purchased from Cell Signaling Technology, Inc. $(1: 2,000)$.

Statistical analysis. All experiments were repeated at least three times. Data were presented as the mean \pm standard deviation. The differences between groups were analyzed with Student's t-test or one-way analysis of variance using SPSS 19.0 software (IBM, Armonk, NY, USA). P<0.05 was considered to indicate a statistically significant difference.

\section{Results}

Baicalein inhibits the motility of AGS human GC cells. The effect of baicalein on cell motility was tested by performing a wound-healing assay. Confluent monolayers of cells were scraped to form a wound and then stimulated with 25 or $50 \mu \mathrm{M}$ baicalein for $24 \mathrm{~h}$. Fig. 1 shows the effect of the treatment with baicalein after $24 \mathrm{~h}$. Treatment with 25 or $50 \mu \mathrm{M}$ baicalein inhibited the wound width in AGS tissue by up to 45.3 or $64.0 \%$ respectively, suggesting that baicalein significantly inhibits AGS cell motility (Fig. 1B).

Baicalein inhibits AGS cell migration and invasion. A Transwell chamber coated with or without Matrigel was employed to respectively study the migratory or invasive capacity of AGS cells. As shown in Fig. 2A, the number of cells that migrated or invaded into the lower chamber was significantly reduced by baicalein treatment in a dose-dependent manner. Quantification analysis indicated that treatment with 25 or $50 \mu \mathrm{M}$ baicalein reduced the migration of AGS by 32.8 and $65.4 \%$, respectively, as well as inhibiting cell invasion by 39.7 and $62.6 \%$, respectively (Fig. 2B).

Baicalein inhibits the TGF- $\beta /$ Smad4 signaling pathway in AGS cells. To investigate the possible mechanisms mediating the anti-metastatic activities of baicalein, qPCR and western blot analyses were performed to examine its effect on the expression of TGF- $\beta$ and Smad4. The mRNA and protein 
A

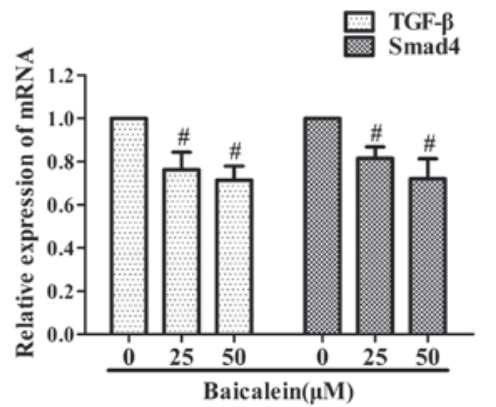

B

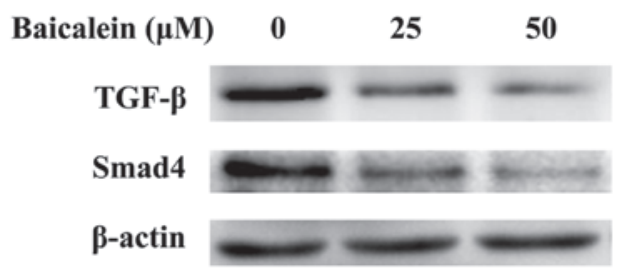

Figure 3. Inhibitory effect of baicalein on autocrine transforming growth factor- $\beta$ (TGF- $\beta$ ) signaling in AGS cells. (A) The mRNA levels of TGF- $\beta$ and Smad4 were determined by quantitative polymerase chain reaction. GAPDH was used as an internal control. Data are represented as the mean \pm standard deviation of three separate experiments. ${ }^{*} \mathrm{P}<0.05$ compared with untreated cells. (B) The protein levels of TGF- $\beta$ and Smad4 were determined by western blotting. $\beta$-actin was used as an internal control. Data are representative of three separate experiments.

expression levels of TGF- $\beta$ and Smad4 were markedly downregulated by baicalein when compared with those of the controls (Fig. 3).

Baicalein reduces the expression levels of $N$-cadherin, vimentin, ZEB1 and ZEB2 in AGS cells. Subsequently, the effect of baicalein on the expression levels of metastasis-associated $\mathrm{N}$-cadherin, vimentin, ZEB1, ZEB2 was investigated. These are critical downstream target genes of the TGF- $\beta$ signaling pathway. As shown in Fig. 4, baicalein treatment markedly decreased the expression of $\mathrm{N}$-cadherin, vimentin, ZEB1 and ZEB2, at the transcriptional and translational levels.

\section{Discussion}

In the majority of GC cases, local invasion and distant metastases are the main causes of treatment failure and mortality. Therefore, there is an urgent requirement to develop novel and more effective strategies for preventing and treating the metastatic stage of this disease. Baicalein, a natural flavonoid extracted from the root of Scutellaria baicalensis, has been considered as a potential anti-metastatic agent for inhibition of cancer cell motility, migration and invasion. It has been demonstrated that baicalein exerts its anti-tumor activities in multiple types of cancer through modulation of multiple signaling pathways $(10,11,13)$. However, the molecular mechanisms by which baicalein exerts its anti-metastatic effects are yet to be elucidated.

The present study used the AGS human GC cell line to demonstrate that baicalein was able to inhibit cell motility, migration and invasion. To investigate the molecular mechanism underlying these effects of baicalein on AGS cells,
A

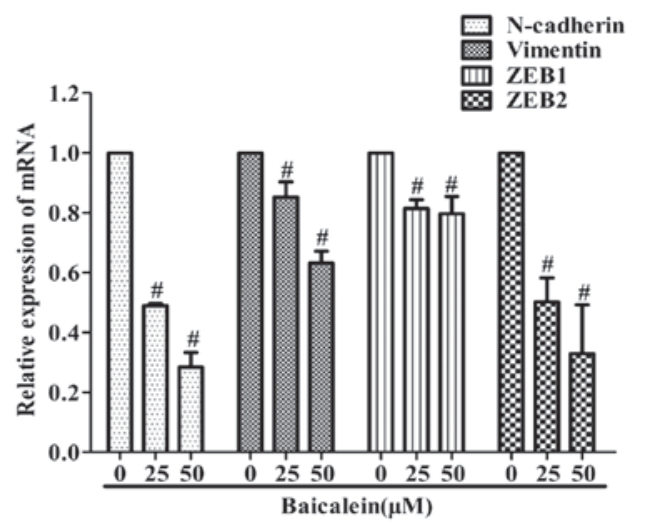

B

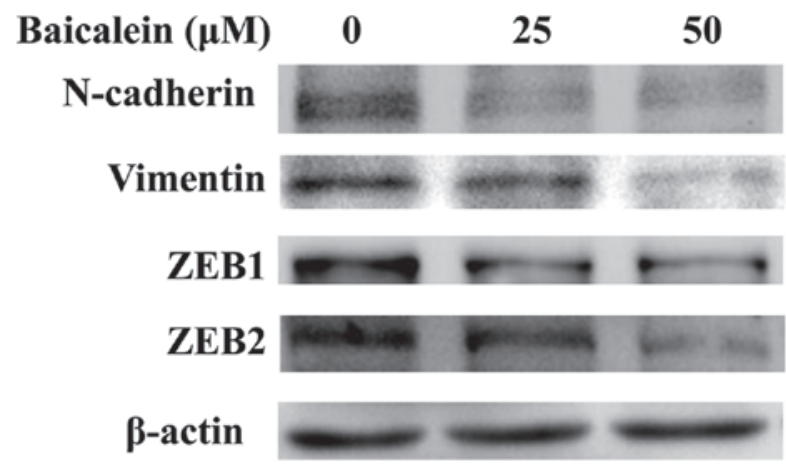

Figure 4. Inhibitory effect of baicalein on the expression of $\mathrm{N}$-cadherin, vimentin, ZEB1 and ZEB2 in AGS cells. The mRNA levels of N-cadherin, vimentin, ZEB1 and ZEB2 were determined by quantitative polymerase chain reaction. GAPDH was used as an internal control. Data are represented as the mean \pm standard deviation of three separate experiments. ${ }^{\#} \mathrm{P}<0.05 \mathrm{com}$ pared with untreated cells. (B) The protein levels of N-cadherin, vimentin, ZEB1 and ZEB2 were determined by western blotting. $\beta$-actin was used as an internal control. Data are representative of three separate experiments.

the alteration of TGF- $\beta$ signaling was analyzed. TGF- $\beta$ is a multifunctional cytokine with dual roles in cancer progression. In the early stages of cancer, it serves a tumor suppressing function either by inhibiting cellular proliferation or by promoting cellular apoptosis. However, in the later stages of cancer, cancer cells become resistant to the growth inhibitory activity of TGF- $\beta$ and even overexpress TGF- $\beta$, which promotes invasion and metastasis by stimulating cell motility, tumor angiogenesis and inducing immunosuppression. Disruption of the tumor autocrine TGF- $\beta$ signaling has been found to inhibit metastasis $(15,16)$. Among the signaling molecules, Smad proteins exhibit a central role in the manifestation of the biological activities of TGF- $\beta$. Smad proteins are classified into three subtypes: Receptor-regulated Smads (R-Smads), common-partner Smads (Co-Smads), and inhibitory Smads (I-Smads). Numerous studies have demonstrated that Smad4, the only Co-Smad in mammals, is essential for TGF- $\beta$-induced tumor cell invasion (17-20). The present study revealed that baicalein inhibits TGF- $\beta$ and Smad 4 expression at the mRNA and protein levels. Thus, it is possible that baicalein may suppress GC cell invasion and metastasis through the inhibition of TGF- $\beta /$ Smad 4 signaling pathway.

Several studies have shown that TGF- $\beta$ signaling enhances cancer cell migration and invasion through multiple adhe- 
sion molecules, cytoskeletal proteins and transcriptional factors (21-23). N-cadherin is a calcium-dependent cell adhesion molecule which functions as an invasion promoter in cancer. Expression of $\mathrm{N}$-cadherin renders cells more motile and invasive (24). Vimentin is a type III mesenchymal filament, a mammalian structural cytoskeletal protein with elevated and aberrant expression that correlates well with upregulated cell invasion and migration in malignancy (25). ZEB1 and ZEB2 are members of the zinc finger E-box binding transcriptional factor family and have been found to be upregulated in GC. Knockdown of ZEB1 and ZEB2 reduces GC cell migration and invasion (26-28).

The downstream signaling targets of TGF- $\beta$ were investigated in the present study to deduce which were involved in the anti-metastastic effects of baicalein on AGS cells. It was revealed that the expression levels of $\mathrm{N}$-cadherin, vimentin, ZEB1 and ZEB2 in AGS cells were repressed by baicalein treatment, suggesting that baicalein may counteract the invasive and metastatic phenotype partly via TGF- $\beta /$ Smad4-induced downregulation of N-cadherin, vimentin, ZEB1 and ZEB2.

In conclusion, this study identified that baicalein modulates TGF- $\beta$, and Smad4 expression levels, as well as N-cadherin, vimentin, ZEB1 and ZEB2 expression levels, which may have inhibited the motility, migration and invasion of AGS cells. These results suggest that baicalein may be a potential anticancer agent for prevention and treatment of GC metastasis. Further studies in clinically relevant animal models should be conducted to exploit its potential benefits against metastatic GC in future.

\section{Acknowledgements}

This study was sponsored by the Key Clinical Specialty Discipline Construction Program of Fujian, China and the Special Funds of the Finance Department of Fujian Province (2012B013)

\section{References}

1. Jemal A, Bray F, Center MM, Ferlay J, Ward E and Forman D: Global cancer statistics. CA Cancer J Clin 61: 69-90, 2011.

2. Hartgrink HH, Jansen EP, van Grieken NC and van de Velde CJ: Gastric cancer. Lancet 374: 477-490, 2009.

3. Derynck R and Akhurst RJ: Differentiation plasticity regulated by TGF-beta family proteins in development and disease. Nat Cell Biol 9: 1000-1004, 2007.

4. Saito H, Tsujitani S, Oka S, et al: The expression of transforming growth factor-betal is significantly correlated with the expression of vascular endothelial growth factor and poor prognosis of patients with advanced gastric carcinoma. Cancer 86: 1455-1462, 1999.

5. Maehara Y, Kakeji Y, Kabashima A, et al: Role of transforming growth factor-beta 1 in invasion and metastasis in gastric carcinoma. J Clin Oncol 17: 607-614, 1999.

6. Ijichi H, Ikenoue T, Kato N, et al: Systematic analysis of the TGF-beta-Smad signaling pathway in gastrointestinal cancer cells. Biochem Biophys Res Commun 289: 350-357, 2001.

7. Wang KS, Hu ZL, Li JH, Xiao DS and Wen JF: Enhancement of metastatic and invasive capacity of gastric cancer cells by transforming growth factor-beta1. Acta Biochim Biophys Sin (Shanghai) 38: 179-186, 2006.
8. Parajuli P, Joshee N, Rimando AM, Mittal S and Yadav AK: In vitro antitumor mechanisms of various Scutellaria extracts and constituent flavonoids. Planta Med 75: 41-48, 2009.

9. Li-Weber M: New therapeutic aspects of flavones: the anticancer properties of Scutellaria and its main active constituents Wogonin, Baicalein and Baicalin. Cancer Treat Rev 35: 57-68, 2009.

10. Wang L, Ling Y, Chen Y, et al: Flavonoid baicalein suppresses adhesion, migration and invasion of MDA-MB-231 human breast cancer cells. Cancer Lett 297: 42-48, 2010.

11. Wu B, Li J, Huang D, et al: Baicalein mediates inhibition of migration and invasiveness of skin carcinoma through Ezrin in A431 cells. BMC Cancer 11: 527, 2011.

12. Wu JY, Tsai KW, Li YZ, et al: Anti-bladder-tumor effect of baicalein from Scutellaria baicalensis Georgi and its application in vivo. Evid Based Complement Alternat Med 2013: 579751, 2013.

13. Chiu YW, Lin TH, Huang WS, et al: Baicalein inhibits the migration and invasive properties of human hepatoma cells. Toxicol Appl Pharmacol 255: 316-326, 2011.

14. Zhang Y, Song L, Cai L, Wei R, Hu H and Jin W: Effects of baicalein on apoptosis, cell cycle arrest, migration and invasion of osteosarcoma cells. Food Chem Toxicol 53: 325-333, 2013.

15. Picon A, Gold LI, Wang J, Cohen A and Friedman E: A subset of metastatic human colon cancers expresses elevated levels of transforming growth factor beta1. Cancer Epidemiol Biomarkers Prev 7: 497-504, 1998.

16. Fransvea E, Angelotti U, Antonaci S and Giannelli G: Blocking transforming growth factor-beta up-regulates E-cadherin and reduces migration and invasion of hepatocellular carcinoma cells. Hepatology 47: 1557-1566, 2008.

17. Takano S, Kanai F, Jazag A, et al: Smad4 is essential for down-regulation of E-cadherin induced by TGF-beta in pancreatic cancer cell line PANC-1. J Biochem 141: 345-351, 2007.

18. Wiercinska E, Naber HP, Pardali E, van der Pluijm G, van Dam $H$ and ten Dijke P: The TGF- $\beta /$ Smad pathway induces breast cancer cell invasion through the up-regulation of matrix metalloproteinase 2 and 9 in a spheroid invasion model system. Breast Cancer Res Treat 128: 657-666, 2011.

19. Deckers M, van Dinther M, Buijs J, et al: The tumor suppressor Smad4 is required for transforming growth factor beta-induced epithelial to mesenchymal transition and bone metastasis of breast cancer cells. Cancer Res 66: 2202-2209, 2006.

20. Shiou SR, Datta PK, Dhawan P, et al: Smad4-dependent regulation of urokinase plasminogen activator secretion and RNA stability associated with invasiveness by autocrine and paracrine transforming growth factor-beta. J Biol Chem 281: 33971-33981, 2006.

21. Araki K, Shimura T, Suzuki H, et al: E/N-cadherin switch mediates cancer progression via TGF- $\beta$-induced epithelial-to-mesenchymal transition in extrahepatic cholangiocarcinoma. Br J Cancer 105: 1885-1893, 2011.

22. Cheng JC, Auersperg N and Leung PC: TGF-beta induces serous borderline ovarian tumor cell invasion by activating EMT but triggers apoptosis in low-grade serous ovarian carcinoma cells. PLoS One 7: e42436, 2012.

23. Dai M, Al-Odaini AA, Fils-Aimé N, et al: Cyclin D1 cooperates with $\mathrm{p} 21$ to regulate TGF $\beta$-mediated breast cancer cell migration and tumor local invasion. Breast Cancer Res 15: R49, 2013.

24. Derycke LD and Bracke ME: N-cadherin in the spotlight of cell-cell adhesion, differentiation, embryogenesis, invasion and signalling. Int J Dev Biol 48: 463-476, 2004.

25. Satelli A and Li S: vimentin in cancer and its potential as a molecular target for cancer therapy. Cell Mol Life Sci 68: 3033-3046, 2011

26. Jia B, Liu H, Kong Q and Li B: Overexpression of ZEB1 associated with metastasis and invasion in patients with gastric carcinoma. Mol Cell Biochem 366: 223-229, 2012.

27. Okugawa Y, Toiyama Y, Tanaka K, et al: Clinical significance of Zinc finger E-box Binding homeobox 1 (ZEB1) in human gastric cancer. J Surg Oncol 106: 280-285, 2012.

28. Dai YH, Tang YP, Zhu HY, et al: ZEB2 promotes the metastasis of gastric cancer and modulates epithelial mesenchymal transition of gastric cancer cells. Dig Dis Sci 57: 1253-1260, 2012. 